\title{
Robotic partial intersphincteric resection with colonic J-pouch anal anastomosis for a very low rectal cancer
}

\author{
E. Gorgun ${ }^{1}$ - C. Benlice ${ }^{1}$
}

Received: 9 May 2016/Accepted: 30 May 2016/Published online: 22 June 2016

(C) Springer-Verlag Italia Srl 2016

In this video, we demonstrate robotic intersphincteric resection of a very low rectal cancer with establishment of intestinal continuity by hand-sewn colonic J-pouch anal anastomosis. This 53-year-old female with recently diagnosed with locally advanced very low rectal moderately differentiated adenocarcinoma and subsequently underwent 6 weeks of neoadjuvant chemoradiotherapy. Consent was obtained verbally for operation. For the resection, a $12-\mathrm{mm}$ camera port was placed at the umbilicus, and three $8-\mathrm{mm}$ robotic ports and additional two $5 \mathrm{~mm}$ were utilized for dissection and retraction. Once patient was positioned, the inferior mesenteric artery was identified and ligated; followed by medial to lateral approach, inferior mesenteric vein was identified and ligated. Subsequently, through the avascular plane, mesorectum was dissected totally down to the level of the pelvic floor with attention paid to not injure the ureter. Dissection was carried into the intersphincteric groove, and the puborectalis muscle was visualized from the end luminal part. This stage was confirmed by digital examination. Once the completely mobilization of the colon was achieved, intersphincteric dissection was started through the perineum. Mucosal incision was made just at the dentate line. Dissection between the external and internal sphincter was carried and reached proximally into the plane from the top. Ultimately, all the connections were freed up and the rectum was exteriorized by using wound protector. Bowel continuity was restored by an intracorporeal double-stapled colonic J-pouch anal anastomosis (video). Finally, decision was made, for functional purposes, to give the patient a diverting loop ileostomy. Patient had an uneventful recovery and was discharged on postoperative day three. Final pathology showed no residual tumor in the bowel wall and 0/13 nodes were involved. As a conclusion, robotic intersphincteric resection is technically feasible and safe for patients with low-lying rectal cancer. This approach provides a deeper dissection in the pelvic cavity with less technical difficulties.

Compliance with ethical standards

Conflict of interest The authors declare that they have no conflict of interest.

Ethical approval For this type of study ethical approval is not necessary.

Informed consent Informed consent was obtained from the patient.
Electronic supplementary material The online version of this article (doi:10.1007/s10151-016-1500-0) contains supplementary material, which is available to authorized users.

\section{E. Gorgun}

gorgune@ccf.org

1 Department of Colorectal Surgery, Digestive Disease Institute, Cleveland Clinic, 9500 Euclid Ave. A-30, Cleveland, OH 44195, USA 